\title{
The Relationship between Bus Drivers' Improper Driving Behaviors and Abnormal Vehicle States Based on Advanced Driver Assistance Systems in Naturalistic Driving
}

\author{
Tao Wang $\mathbb{D}^{1},{ }^{1}$ Yuzhi Chen $\mathbb{D}^{1},{ }^{1}$ Xingchen Yan $\mathbb{D}^{2},{ }^{2}$ Jun Chen $\mathbb{D},{ }^{3}$ and Wenyong Li $^{1}{ }^{1}$ \\ ${ }^{1}$ School of Architecture and Transportation, Guilin University of Electronic Technology, Guilin 541004, China \\ ${ }^{2}$ Collage of Automobile and Traffic Engineering, Nanjing Forestry University, Longpan Road 159\#, Nanjing 210037, China \\ ${ }^{3}$ School of Transportation, Southeast University, Dongnandaxue Road 2\#, Jiangning Development Zone, Nanjing 211189, China \\ Correspondence should be addressed to Tao Wang; wangtao@seu.edu.cn
}

Received 6 June 2020; Revised 19 July 2020; Accepted 23 July 2020; Published 20 August 2020

Academic Editor: Peter Dabnichki

Copyright (c) 2020 Tao Wang et al. This is an open access article distributed under the Creative Commons Attribution License, which permits unrestricted use, distribution, and reproduction in any medium, provided the original work is properly cited.

In order to improve the adaptation of driver to the advanced driver assistance system (ADAS) and optimize the active safety control technology of vehicle under man-computer cooperative driving, this paper investigated the correlation between driver's improper driving behaviors and abnormal vehicle states under the ADAS. Based on the warning data collected from the driver's assistance warning system equipped on buses, the interaction between improper behaviors, between abnormal vehicle states, and between improper behaviors and abnormal vehicle states were quantitatively analyzed through the hierarchical clustering method and improved Apriori algorithm. The results showed that eye closure and yawn were high in concurrency (probability: 0.888) and interaction (average probability: 0.946); the interaction among lane departure, rapid acceleration, and rapid deceleration are frequent (average probability: 0.7224); eye closure (average probability: 0.452 ) and yawn (average probability: 0.444) are likely to induce abnormal vehicle states such as rapid acceleration and rapid deceleration. Some suggestions proposed based on the results are as follows. First, it is suggested that the ADAS should combine the warning modes of eye closure and yawn; second, when the driver closes eyes or yawns, the control of the ADAS over the lateral and longitudinal performance of vehicle should be enhanced; third, the extent of control by the ADAS should be determined according to the relationship probability; finally, the lateral control over the vehicle by the ADAS should be strengthened when there is a forward collision warning.

\section{Introduction}

The . Department of Transportation, SAE, etc. [1] classified the development of the intelligent vehicle into six levels of no automation, driver assistance, partial automation, conditional automation, high automation, and full automation. Although different levels and functions of intelligent vehicle technology are developing rapidly, the real sense of full working condition automatic driving has been difficult to achieve in the short term [2]. We have only entered the initial stage of man-computer cooperative driving where drivers and automatic driving systems collaborate with each other and will be in this phase for a long time. Man-computer collaborative driving means that under nonfully automated driving conditions, the driver and the vehicle's intelligent control system can cooperatively complete driving in the loop. And how to realize the hybrid enhancement of the man-computer system to avoid causing man-computer conflict and nonco-operative mode is the most important key to improve the efficiency of mancomputer cooperative driving key factors [3].

However, interaction of automated vehicle and human driver is not a well-studied subject [4]. During man-computer cooperative driving, the automated vehicle and the driver can be described as two entities that perform control together [5]. In this regard, many scholars often study both the driving behavior of the driver and the state of the vehicle to facilitate the driver's and the intelligent vehicle's efficient cooperation. Sun et al. [6] analyzed driver lane change behavior characteristics and proposed an adaptive algorithm 
for estimating the optimal threshold of lane change warning (LCW) in real time to improve the performance of the LCW system. Zhu et al. [7] analyzed the driver's lane change time and lane change behavior characteristics under typical conditions using driving simulators and proposed a personalized vehicle lane change assistance system that combined driver behavior recognition strategies. Sang et al. [8] determined the collision risk based on the driver's driving characteristics and proposed a collision warning system based on the individual driver's driving behavior. Wang et al. [9] determined different time-to-collision (TTC) warning thresholds based on different driving styles. Ahmadi and Machiani [10] considered the driver perceived reaction time (PRT) factor and adjusted the warning time in real time using the reward and punishment function. And an adaptive curve speed warning system (ACSW) was designed.

Vehicle performance and safety are directly related to the control measures taken by the driver as the adaptive, optimal decision controller of the vehicle. Among them, the estimation of the vehicle state is an important issue in vehicle dynamics and is one of the key technologies to achieve an active safety control system for the vehicle [11]. Therefore, there has also been consideration of vehicle condition factors to improve advanced driver assistance systems to optimize human-machine interaction. Hsiao et al. [12] considered the vehicle as a polygon in the lane departure warning mechanism and added the vehicle speed information towards the lane boundary to optimize the warning judgment. Sun et al. [13] designed a risk prediction model considering the speed parameters when the vehicle is in the two states of critical skid or rollover. And the warnings of system were also optimized according to the risk level. Many other scholars have used the vibration diagnostic method [14], cyberphysical method [15], unscented Kalman filtering algorithm [16], multiple models [17], and multiple algorithms [18] to optimize the system for accurate estimation of vehicle states and control, considering vehicle state parameters such as rolling bearing state, reliable distribution of vehicle's longitudinal velocity, lateral deviation of vehicle mass center, and tire adhesion.

From the above literature, although optimizing mancomputer cooperation in terms of driver's driving behavior and vehicle state, respectively, has been quite effective. However, they had neglected the influence between driving behavior and vehicle state in the process of man-computer cooperative driving. Li et al. [19] indicated that for intelligent vehicles with advanced driver assistance systems, it is necessary to combine the two directions of driver and system control (the effect of their control is reflected by the vehicle state) into a single integrated system to improve the safety and comfort of the vehicle. Li et al. [20] proposed a driving style estimation method based on maneuver transition probability to promote the optimization of man-computer cooperation, considering multiple vehicle maneuver states (e.g., free driving, approaching, near following, and constrained left and right lane changes). $\mathrm{Na}$ and Cole [21] explored the relationship between driving behavior and vehicle front wheel steering using the Stackelberg equilibrium theory. They also explored the control strategy of driver and active front wheel steering by applying a linear quadratic dynamic optimization algorithm and a distributed model predictive control method, respectively. Zhou et al. [22] considered relevant vehicle state indicators to study the behavior characteristics of driver while driving on the deceleration lane of city expressway ramp. Despite that, they failed to consider the quantification of the relationship between driving behavior and vehicle state. The limitation of these studies lies in that the driver's safety control over the vehicle is studied only from the driving behavior or vehicle state before the warning or accident. Also, the interactive influence of multiple warning occurrences in the ADAS was not considered. In the study of man-computer cooperative driving, we should further investigate the interaction between multiple warnings in the ADAS [23]. The best method for the man-computer cooperative control research, the ADAS as the mainstream equipment of man-computer cooperative control systems nowadays, is to conduct statistical analysis on the actual warning date of vehicles equipped with the common ADAS [24].

Therefore, based on warning data of the driver state monitoring (DSM) system and advanced driver assistance system (ADAS), the relationship between improper driver behaviors and abnormal vehicle states was explored under ADAS conditions, and the interactive influence between multiple warnings was analyzed. So as to provide scientific basis for optimizing the interaction between driver and the ADAS research and promote the development of mancomputer cooperative driving. In this study, the warning data were preprocessed and classified into the corresponding intervals through the hierarchical clustering method for later investigation of the association rules. Then, an improved effectivity-based Apriori algorithm was proposed to explore the association between driver's improper driving behaviors, abnormal vehicle states, and improper driving behaviors and abnormal vehicle states, so as to quantify the correlation between driving behavior and vehicle state.

The rest of this paper was arranged as follows. In the next section, Materials and Methods, we introduced the general situation of data acquisition equipment together with the data selection method and preprocessing process and proposed a relationship mining method based on the improved Apriori algorithm. In Section 3, Results, we verified the existence of the mined relationships and the validity of data clustering results and analyzed the relationship mining results based on the improved Apriori algorithm (including relationship between improper driving behaviors, relationship between abnormal vehicle states, and relationship between improper driving behaviors and abnormal vehicle states). In the final section, Discussion, we discussed the practical significance of the association rules and put forward some suggestions on optimizing the active safety control technology of the ADAS.

\section{Materials and Methods}

2.1. Equipment Overview. In this paper, the warning data of Zhenjiang municipal bus driver's assistance warning system was adopted for the analysis, which can avoid the influence 
on effectiveness of research results brought by the subjectivity of questionnaire and unfaithful environmental simulation of the simulator.

The driver assistance warning system is composed of the driver state monitoring system and ADAS. Its front-end equipment mainly consists of an intelligent driving terminal and a DVR (digital video recorder) vehicle data recorder installed on the bus. The intelligent driving terminal, which contains a DSM camera of the built-in fatigue driving warning system, an ADAS camera, and an active safety warning system loudspeaker, is used to collect the real-time video warning data of the bus driver. The DVR vehicle data recorder, which is composed of a video displayer and two DVR vehicle data recorders, can cooperate with the intelligent driving terminal to realize the real-time data acquisition of radar warning data of the bus and the front vehicle and of the driving characteristics of the bus. The video displayer is used to display the images captured by the camera of the DVR vehicle data recorder. The layout of the equipment is shown in Figure 1.

2.2. Data. In order to ensure the stability and effectivity of the collected warning data as well as the universality of the research results, in this paper, special bus routes such as temporary bus lines, community bus lines, Medicheng circulation lines, and tourism bus lines were excluded. The warning data were collected from the rest 113 bus lines for analysis.

Aiming at the problems such as data redundancy, data exception, data missing, inconsistent data forms, and data matching errors, data cleaning is conducted. The basic data obtained include time, warning type, longitude, latitude, route, warning location, and license plate number. The warning types include eye closure, yawn, and looking away. As for the warning data regarding vehicle state, there are lane deviation, rapid acceleration, rapid deceleration, and forward collision. The warning types and the conditions for triggering the warning system are presented in Table 1.

\subsection{An Improved Apriori Algorithm Based on Effectivity.} Association rule analysis is one of the effective methods for exploring the potential relationship between data items. Among them, the most typical approach is the Apriori algorithm. In this Apriori algorithm, the data are scanned repeatedly to generate the candidate $\mathrm{k}$-item set and calculate the support count; then, the frequent k-item set is selected based on support, so the strong association rules can be extracted based on confidence. This process is repeated until the candidate k-item set is empty, in which case the extraction of association rules is complete, and so the Apriori algorithm is ended.

In the conventional Apriori algorithm, some support (confidence) is slightly smaller than the minimum threshold, but the corresponding confidence (support) is large enough, so that the association rules that can well reflect the relationship between things are lost. It is not feasible to solve this problem by adjusting the minimum threshold given that a large number of redundant rules will be generated. Aiming at this problem, four concepts, namely effectivity, minimum effectivity, ordered k-items set, and ordered frequent k-items set, are introduced as shown below:

Effectivity (Eff):

$$
\text { Eff }=\sqrt{\frac{1}{2}\left(\operatorname{Sup}^{2}+\operatorname{Conf}^{2}\right)}, \quad 0 \leq \text { Eff }<1 .
$$

The minimum effectivity (Mineff):

$$
\text { Mineff }=\sqrt{\frac{1}{2}\left(\text { Minsup }^{2}+\operatorname{Minconf}^{2}\right)}
$$

Ordered $k$-item set: the set containing $k$ items is unordered except for the last item whose order is fixed

Ordered frequent $k$-item set: an ordered k-item set with the effectivity larger than the minimum effectivity

The improved Apriori algorithm flow is shown in Figure 2. This algorithm can not only extract the strong association rules obtained by the traditional Apriori algorithm but also mine the general correlation rules between strong association rules and invalid rules, fully mining the hidden correlation rules in the database. Furthermore, it can avoid the redundant rules in the conventional Apriori algorithm generated by adjusting the minimum support (or confidence) threshold and the aimless adjustment of the minimum support (or confidence) threshold. The minimum optimization degree of the improved Apriori algorithm is calculated to be

$$
\text { Minopti }=\frac{\pi\left(\text { Minsup }^{2}+\text { Minconf }^{2}\right)-\text { Minsup } \cdot \text { Minconf }}{\text { Minsup } \cdot(1-\text { Minconf })+(1-\text { Minsup }) \cdot \text { Minconf }}
$$

where Minopti was the minimum degree of optimization, Minsup is the minimum support, and Minconf is the minimum confidence.

The improved Apriori algorithm proposed based on the introduced validity metrics has the following algorithmic steps:

Step 1. Set thresholds for minimum support (Minsup) and minimum confidence (Minconf), and calculate minimum effectiveness (Mineff) according to actual needs, so that $k=1$

Step 2. Scan the transaction database for a total number of transactions (sets of items) of $N$, and calculate the support count for all 1 set

Step 3. Generate the ordered k-item set and judge whether the ordered k-terms set is empty or not; if it is empty, output all strong association rules and general association rules and end the algorithm; if it is not empty, proceed to the next step

Step 4. Calculate the validity of each ordered k-item set, and extract the ordered $\mathrm{k}$-item set that meets the 


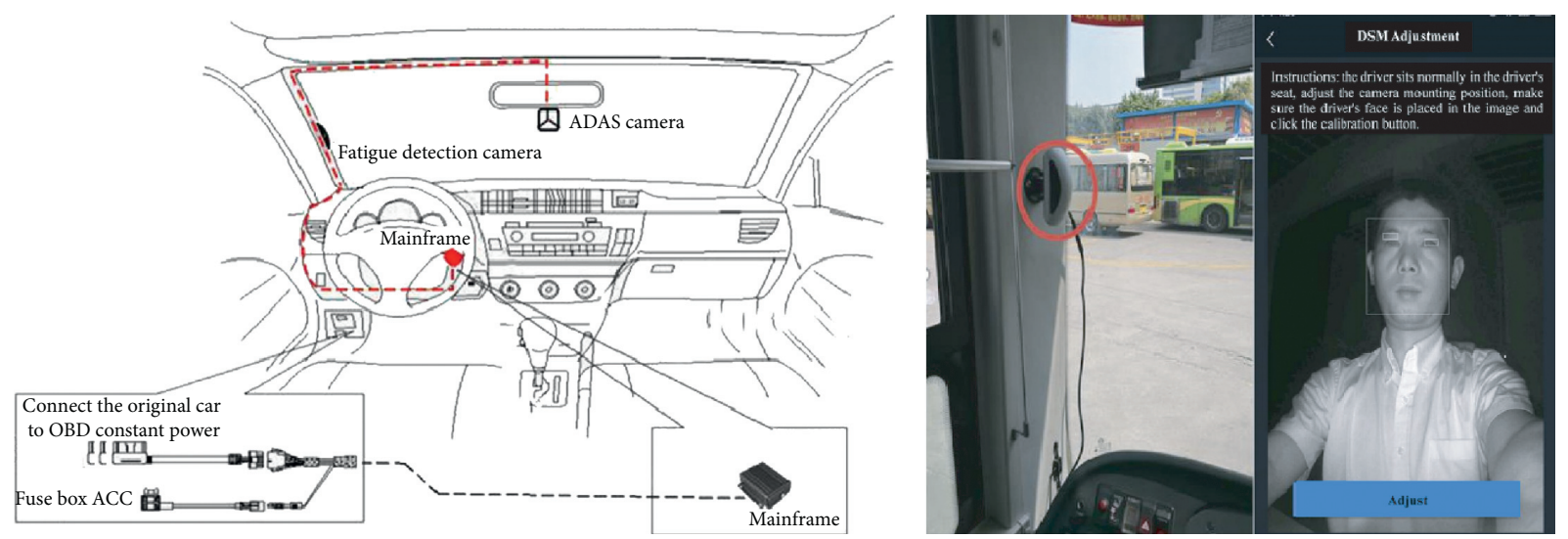

FIgURE 1: Equipment layout.

TABLE 1: Description of the trigger for each type of warning.

\begin{tabular}{|c|c|c|}
\hline Warning type & Condition for triggering the warning system & Sample size \\
\hline Eye closure & $\begin{array}{c}\text { The warning system will be triggered when the driver's eyes keep closed for a long time } \\
\text { while driving. }\end{array}$ & 6414 \\
\hline Yawn & The warning system will be triggered once the driver yawns while driving. & 6665 \\
\hline Looking away & $\begin{array}{c}\text { The warning system will be triggered when the driver keeps looking to the left or right for } \\
\text { over } 5 \mathrm{~s} \text {. }\end{array}$ & 787 \\
\hline Lane departure & $\begin{array}{l}\text { The warning system will be triggered once the vehicle's speed exceeds } 55 \mathrm{k} \mathrm{m} / \mathrm{h} \text { and deviates } \\
\text { from the lane to cross the lane line, except when the vehicle's turn signal is on, the lane } \\
\text { change occurs in acceleration/deceleration state, the vehicle switches lanes rapidly, there is } \\
\text { no line, or the lane lines are not clear. }\end{array}$ & 3457 \\
\hline $\begin{array}{l}\text { Rapid acceleration and rapid } \\
\text { deceleration }\end{array}$ & $\begin{array}{l}\text { The warning system will be triggered once the longitudinal acceleration change value } \\
\text { exceeds the corresponding threshold value prescribed by the SGS safety standard. }\end{array}$ & $\begin{array}{l}1035 \text { and } \\
3146\end{array}$ \\
\hline Forward collision & $\begin{array}{l}\text { The warning system will be triggered once the vehicle's speed exceeds } 55 \mathrm{~km} / \mathrm{h} \text { or when the } \\
\text { vehicle is running at a constant speed of over } 30 \mathrm{~km} / \mathrm{h} \text {, and meanwhile, its relative speed is } \\
\text { faster than the vehicle in the front of it, and the relative time distance (the distance between } \\
\text { the vehicle's head and the tail of its front vehicle/(vehicle speed-speed of the front vehicle)) } \\
\text { is within } 3 \mathrm{~s} \text {. }\end{array}$ & 2360 \\
\hline
\end{tabular}

conditions as the ordered frequent k-item set (note that when $k=1$, the validity is the degree of support, and the minimum validity is equal to the minimum degree of support)

Step 5. Determine whether the conditions are met for an ordered set of frequent $k$ items; if so, the rule from the set of frequent $k$ items is classified as a strong rule; otherwise, the rule from that set of frequent $k$ items is classified as a general rule

Step 6. Connect the ordered frequent k-item set to the ordered frequent 1 -item set, get $N k$-item sets (at this time $k=k+1$ ), and calculate the support count, return to Step 3

\section{Results}

3.1. Data Clustering. To facilitate the use of association rule algorithm to explore the relationship between improper driving behaviors and abnormal vehicle states, the warning data were classified into their corresponding intervals, respectively. The person correlation analysis was conducted on the warning data after cleaning, and the analysis results are shown in Table 2. It could be observed that the correlation coefficient between any two warning types was larger than 0.9 , and the significance level was lower than 0.01 , indicating that the interaction between various warning types was obvious, and thus cluster analysis was feasible.

The unsupervised learning hierarchical clustering analysis and nearest neighbor were used to calculate the between-class distance for cluster analysis. The number of clusters (number of intervals) was 363.

The spatial distribution of the processed warning data was consistent with that of the bus lines, so was their density. The spatial distribution of data is shown in Figure 3. It can be seen from Figure 4 that the intervals with 2 warning items are the most, followed by 3,4 , and 5 items. In contrast, the intervals containing 6 or 7 warning items were the least, 


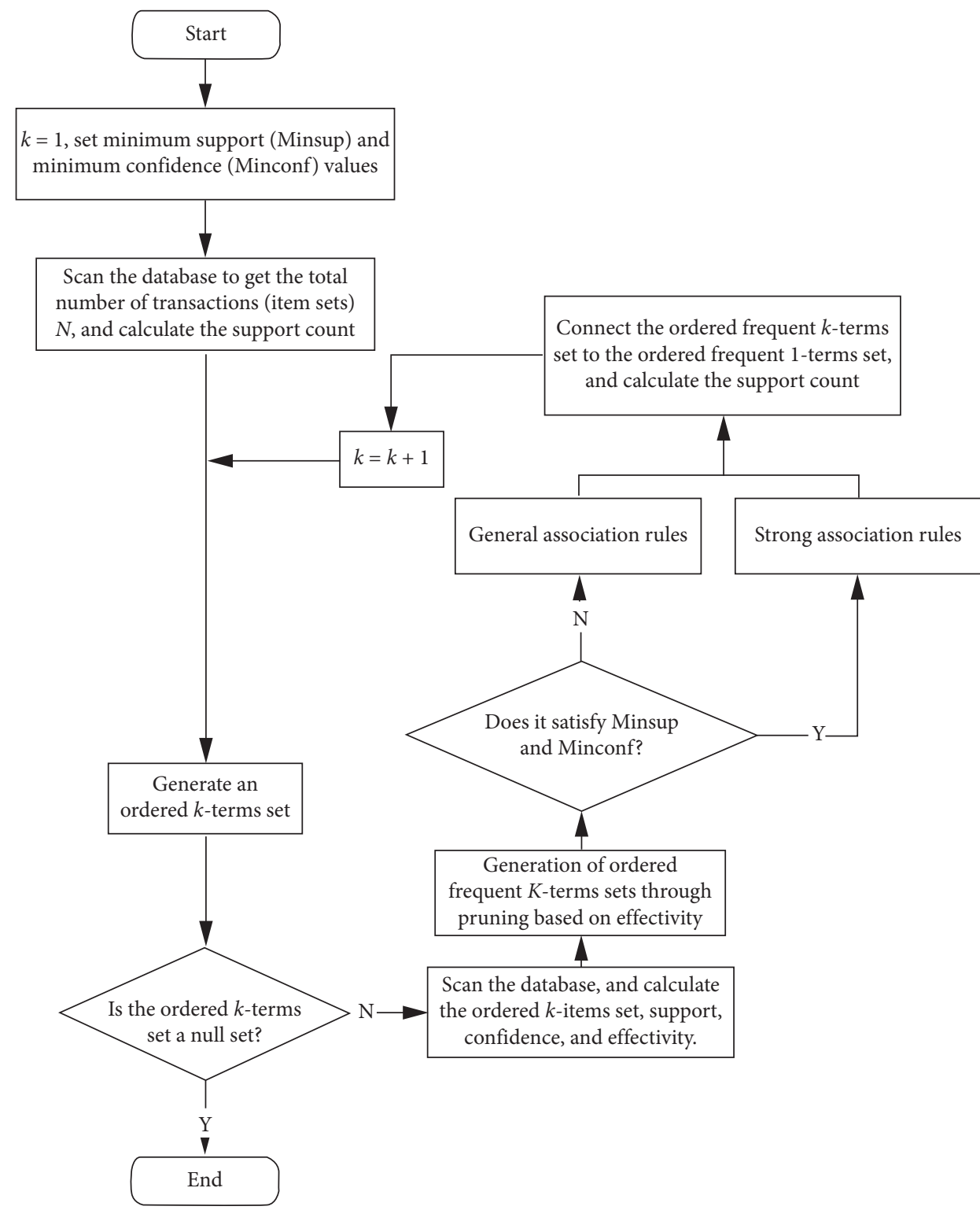

FIGURE 2: The algorithm flowchart of improved Apriori based on effectivity.

which agrees with the occurrence rule of actual warning. After hierarchical clustering, the data were reliable, and analysis can be carried out on association rules.

\subsection{Association Rules between Improper Driving Behaviors} and Abnormal Vehicle States. The improved Apriori algorithm was employed to mine the warning data so as to quantify the relationship between driving behavior and vehicle state. After referring to previous experience $[25,26]$ and considering the characteristics of warning data, the minimum support was set to 0.2 and the minimum confidence was set to 0.4 , so the minimum effectivity is 0.316 . The improved Apriori algorithm program was then written in python language for calculation. In view of the actual operating state of bus and the existing experimental conditions, the relationship between improper driving behaviors, between abnormal vehicle states, and between improper driving behaviors and abnormal vehicle state was investigated.

3.2.1. Relationship between Improper Driving Behaviors. The rules of association between improper driving behaviors are given in Table 3 .

It could be seen that the support and confidence between eye closure and yawn were greater than the corresponding minimum thresholds. Thus, "eye closure-yawn" and "yawn-eye closure" were strong rules, indicating that the interaction between eye closure and yawn was significant. The probability of eye closure and yawn where both occur was 0.888 , and the probability of eye closure (yawn) followed by yawn (eye closure) was 0.928 (0.963). The probabilities of looking away occurring with eye closure or yawn were smaller than the minimum support, but the effectivity was larger than the minimum effectivity, so the rule was effective. 
TABle 2: The Person correlation analysis results among the warning types.

\begin{tabular}{|c|c|c|c|c|c|c|c|c|}
\hline \multirow[b]{2}{*}{ Warning types } & \multirow[b]{2}{*}{ Results } & \multicolumn{7}{|c|}{ Warning types } \\
\hline & & $\begin{array}{c}\text { Eye } \\
\text { closure }\end{array}$ & Yawn & $\begin{array}{c}\text { Looking } \\
\text { away }\end{array}$ & $\begin{array}{c}\text { Lane } \\
\text { departure }\end{array}$ & $\begin{array}{c}\text { Rapid } \\
\text { acceleration }\end{array}$ & $\begin{array}{c}\text { Rapid } \\
\text { deceleration }\end{array}$ & $\begin{array}{l}\text { Forward } \\
\text { collision }\end{array}$ \\
\hline \multirow{3}{*}{ Eye closure } & P.R. & 1 & $0.988^{* *}$ & $0.944^{* *}$ & $0.998^{* *}$ & $0.994^{* *}$ & $0.994^{* *}$ & $0.999^{* *}$ \\
\hline & S. & & 0.000 & 0.000 & 0.000 & 0.000 & 0.000 & 0.000 \\
\hline & $\mathrm{N}$ & 2753 & 1585 & 180 & 1153 & 760 & 2316 & 1052 \\
\hline \multirow{3}{*}{ Yawn } & P.R. & $0.988^{* *}$ & 1 & $0.976^{* *}$ & $0.991^{* *}$ & $0.995^{* *}$ & $0.983^{* *}$ & $0.989^{* *}$ \\
\hline & S. & 0.000 & & 0.000 & 0.000 & 0.000 & 0.000 & 0.000 \\
\hline & $\mathrm{N}$ & 1585 & 1585 & 180 & 1153 & 760 & 1585 & 1052 \\
\hline \multirow{3}{*}{ Looking away } & P.R. & $0.944^{* *}$ & $0.976^{* *}$ & 1 & $0.991^{* *}$ & $0.994^{* *}$ & $0.981^{* *}$ & $0.982^{* *}$ \\
\hline & S. & 0.000 & 0.000 & & 0.000 & 0.000 & 0.000 & 0.000 \\
\hline & $\mathrm{N}$ & 180 & 180 & 180 & 180 & 180 & 180 & 180 \\
\hline \multirow{3}{*}{ Lane departure } & P.R. & $0.998^{* *}$ & $0.991^{* *}$ & $0.991^{* *}$ & 1 & $0.997^{* *}$ & $0.996^{* *}$ & $0.999^{* *}$ \\
\hline & S. & 0.000 & 0.000 & 0.000 & & 0.000 & 0.000 & 0.000 \\
\hline & $\mathrm{N}$ & 1153 & 1153 & 180 & 1153 & 760 & 1153 & 1052 \\
\hline \multirow{3}{*}{$\begin{array}{l}\text { Rapid } \\
\text { acceleration }\end{array}$} & P.R. & $0.994^{* *}$ & $0.995^{* *}$ & $0.994^{* *}$ & $0.997^{* *}$ & 1 & $0.993^{* *}$ & $0.992^{* *}$ \\
\hline & $\mathrm{S}$. & 0.000 & 0.000 & 0.000 & 0.000 & & 0.000 & 0.000 \\
\hline & $\mathrm{N}$ & 760 & 760 & 180 & 760 & 760 & 760 & 760 \\
\hline \multirow{3}{*}{$\begin{array}{l}\text { Rapid } \\
\text { deceleration }\end{array}$} & P.R. & $0.994^{* *}$ & $0.983^{* *}$ & $0.981^{* *}$ & $0.996^{* *}$ & $0.993^{* *}$ & 1 & $0.998^{* *}$ \\
\hline & S. & 0.000 & 0.000 & 0.000 & 0.000 & 0.000 & & 0.000 \\
\hline & $\mathrm{N}$ & 2316 & 1585 & 180 & 1153 & 760 & 2316 & 1052 \\
\hline \multirow{3}{*}{$\begin{array}{l}\text { Forward } \\
\text { collision }\end{array}$} & P.R. & $0.999^{* *}$ & $0.989^{* *}$ & $0.982^{* *}$ & $0.999^{* *}$ & $0.992^{* *}$ & $0.998^{* *}$ & 1 \\
\hline & S. & 0.000 & 0.000 & 0.000 & 0.000 & 0.000 & 0.000 & \\
\hline & $\mathrm{N}$ & 1052 & 1052 & 180 & 1052 & 760 & 1052 & 1052 \\
\hline
\end{tabular}

P.R. is Pearson correlation; S. denotes significant (two-tailed); $\mathrm{N}$ denotes the degree of freedom; ${ }^{* *}$ indicates that the correlation is significant at a significance level (double test) less than 0.01 .

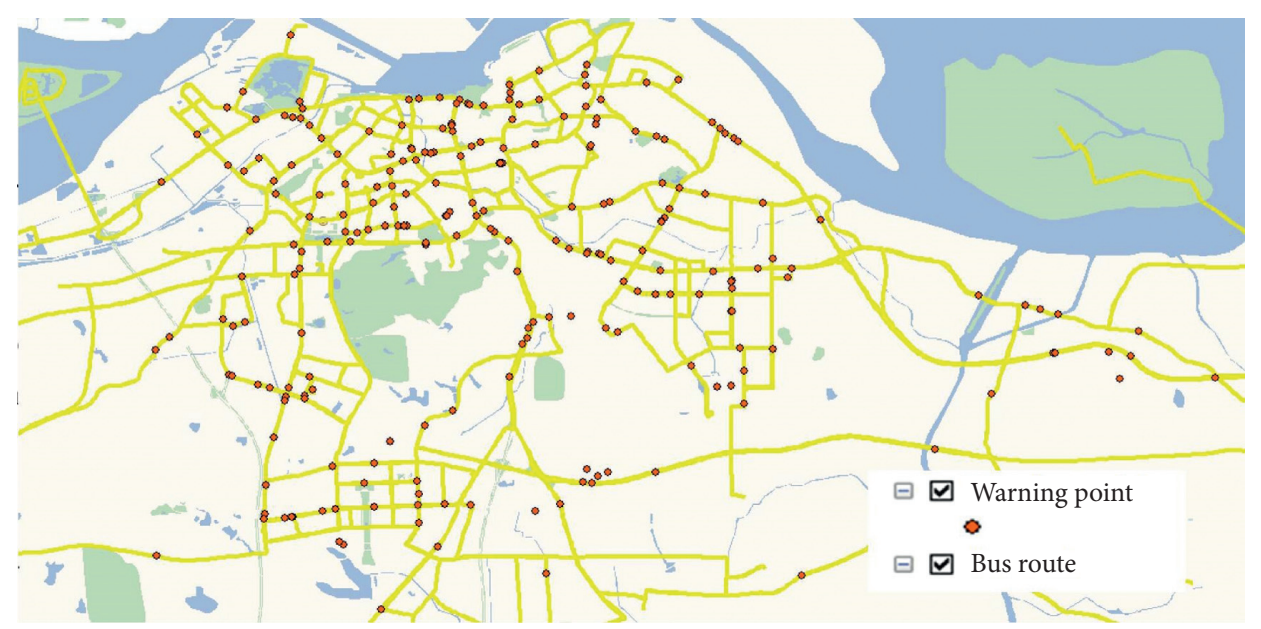

FIGURE 3: Spatial and temporal distribution of warning data after clustering processing.

The probabilities of looking away followed by eye closure and yawn were, respectively, 0.961 and 0.922. Similarly, the probability of looking away and yawn (eye closure) followed by eye closure (yawn) was 0.957 (0.918).

The spatial and temporal distributions of eye closure and yawn are shown in Figure 5 where the vertical axis was the spatial data obtained by dimensionality reduction of the latitude and longitude data, and the horizontal axis was the time data obtained by standardizing the warning occurrence time data. Figure 5 shows that the spatial and temporal distributions of eye closure and yawn are roughly consistent, and the two centers of mass are close to each other, indicating that the warning data used for association rule mining are effective and reliable. The cumulative degree of interactions between eye closure, yawn, and looking away is illustrated in Figure 6. The number of interactions between eye closure and yawn was significantly higher than that between eye closure and looking away, as well as that between yawn and looking away. This result was consistent with the association rules, indicating that the association rules were effective and reliable.

3.2.2. Relationship between Abnormal Vehicle States. The association rules between abnormal vehicle states obtained are given in Table 4 . The association rules among lane 


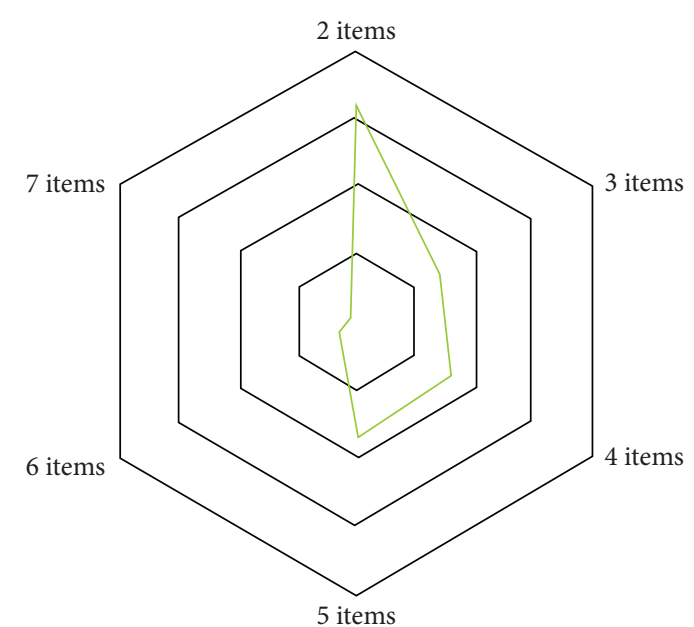

Figure 4: Distribution of the number of warning items.

TABLE 3: Association rules for improper driving behaviors.

\begin{tabular}{lccc}
\hline Association rules & Support & Confidence & Effectivity \\
\hline Eye closure-yawn* & 0.888 & 0.928 & 0.908 \\
Yawn-eye closure* & 0.888 & 0.963 & 0.926 \\
Looking away-eye closure & 0.140 & 0.961 & 0.687 \\
$\begin{array}{l}\text { Looking away-yawn } \\
\text { Looking away-yawn-eye }\end{array}$ & 0.135 & 0.922 & 0.659 \\
$\begin{array}{l}\text { closure } \\
\begin{array}{l}\text { Looking away-eye } \\
\text { closure-yawn }\end{array}\end{array}$ & 0.129 & 0.957 & 0.683 \\
\hline
\end{tabular}

Note: *indicates strong rules; otherwise, they are general rules.

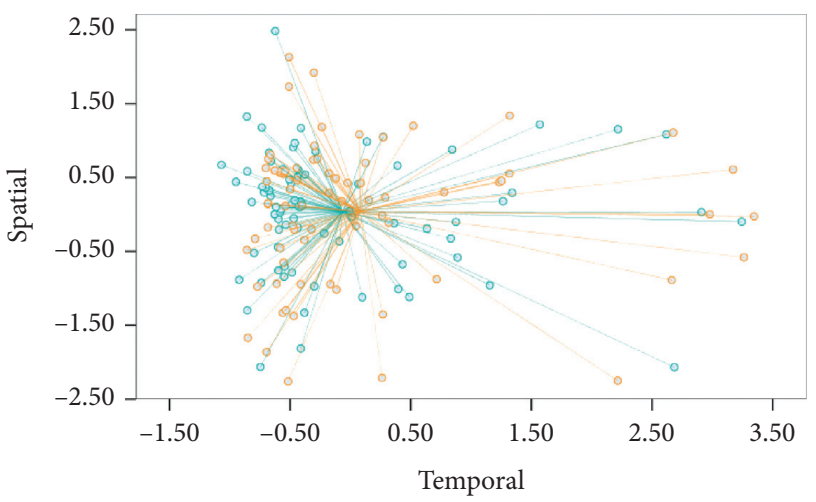

$$
\begin{aligned}
& \text { Warning type } \\
& \text { - }- \text { Eye closure } \\
& -\mathrm{-} \text { Yawn }
\end{aligned}
$$

Figure 5: Spatial and temporal distributions of eye closure and yawn.

departure, rapid acceleration, and rapid deceleration were all strong rules, and they interacted with each other remarkably. The occurrence probabilities of any two or all three were higher than the minimum support, 0.2. The probabilities of lane departure followed by rapid acceleration and rapid deceleration were 0.763 and 0.675 , respectively. The probabilities of rapid deceleration followed by lane departure and

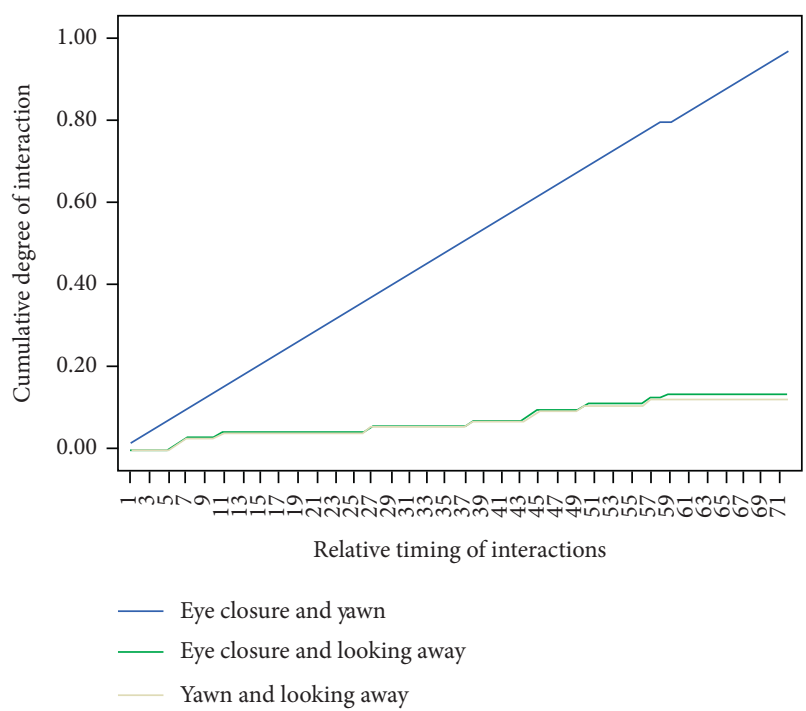

Figure 6: Cumulative interaction among eye closure, yawn, and looking away.

rapid acceleration were 0.507 and 0.875 , respectively. The occurrence probability of rapid acceleration followed by rapid deceleration was 0.792 , indicating a strong interaction between rapid acceleration and rapid deceleration.

The probability of rapid acceleration and lane departure followed by rapid deceleration was 0.874 , while the probability of rapid deceleration and lane departure followed by rapid acceleration was 0.987 , as compared against 0.571 , the probability of rapid deceleration and rapid acceleration followed by lane departure. The probability of forward collision and lane departure where both occur was smaller than the minimum support, but the probability of forward collision followed by lane departure was 1.000 , much higher than the minimum effectivity.

Figure 7 shows the distribution of various warning types, from which it can be seen that the distributions of lane departure, rapid acceleration, and rapid deceleration are roughly the same, and the three centers of mass are close to each other, indicating that the warning data used for association rule mining are effective and reliable. The interaction among rapid deceleration, rapid acceleration, and lane departure is shown in Figure 8 where the horizontal axis represents the sequence of warnings at the time of occurrence, vertical axis represents the warning type (1 stands for lane departure, 2 for rapid acceleration, and 3 for rapid deceleration), and the numbers in the legend are the interval numbers. By connecting the warning points, the lines in Figure 8 are formed, from which the interaction between the points could be observed. The interaction among lane departure and rapid acceleration and rapid deceleration was frequent, among which that between rapid acceleration and rapid deceleration was the most significant, which agreed with the association rules, indicating that the association rules were reliable.

3.2.3. Relationship between Improper Driving Behaviors and Abnormal Vehicle States. The association rules are listed in Table 5. The association rules among eye closure, yawn, rapid 
TABLE 4: Association rules among various abnormal vehicle states.

\begin{tabular}{|c|c|c|c|}
\hline Association rules & Support & Confidence & Effectivity \\
\hline Lane departure-rapid acceleration* & 0.249 & 0.763 & 0.568 \\
\hline Lane departure-rapid deceleration* & 0.221 & 0.675 & 0.502 \\
\hline Rapid acceleration-lane departure-rapid deceleration* & 0.218 & 0.874 & 0.637 \\
\hline Rapid acceleration-rapid deceleration* ${ }^{*}$ & 0.381 & 0.792 & 0.621 \\
\hline Rapid deceleration-lane departure* & 0.221 & 0.507 & 0.391 \\
\hline Rapid deceleration-rapid acceleration-lane departure* & 0.218 & 0.987 & 0.715 \\
\hline Rapid deceleration-rapid acceleration* ${ }^{*}$ & 0.381 & 0.875 & 0.675 \\
\hline Rapid deceleration-rapid acceleration-lane departure* & 0.218 & 0.571 & 0.432 \\
\hline Forward collision-lane departure & 0.109 & 1.000 & 0.711 \\
\hline
\end{tabular}

Note: *indicates strong rules; otherwise, they are general rules.

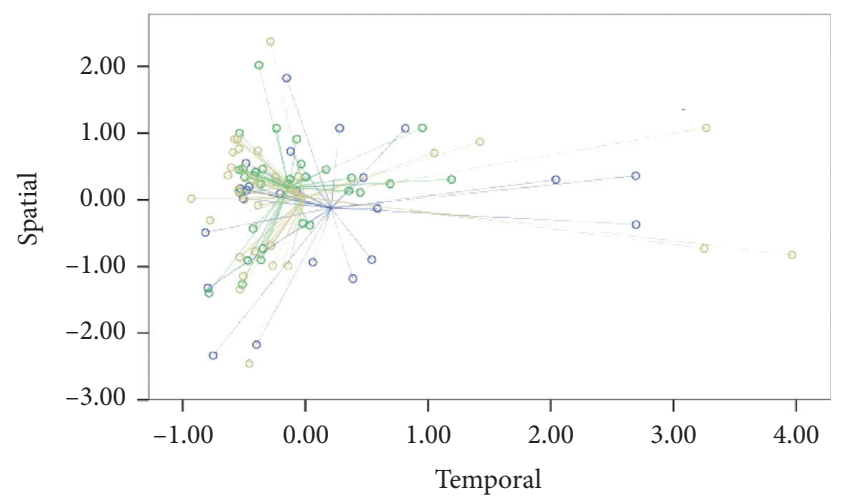

Warning type

- Lane departure

- Rapid acceleration

- Rapid deceleration

FIGURE 7: Spatial and temporal distribution of lane departure, rapid acceleration, and rapid deceleration.

acceleration, and rapid deceleration were all strong rules, indicating that they were the major influencing indicators in interaction between improper driving behaviors and abnormal vehicle states. The probabilities of rapid acceleration, rapid deceleration, and lane departure induced by eye closure were $0.476,0.428$, and 0.335 , respectively. The probabilities of rapid acceleration, rapid deceleration, and lane departure induced by yawn were $0.481,0.407$, and 0.354 , respectively. The probabilities of rapid acceleration, rapid deceleration, and lane departure induced by eye closure and yawn together were $0.481,0.403$, and 0.361 , respectively. The probabilities of rapid acceleration and rapid deceleration induced by yawn and lane departure together were 0.763 and 0.675 , respectively.

The spatial and temporal distributions of eye closure, yawn, lane departure, rapid acceleration, and rapid deceleration were roughly consistent. Moreover, the mass centers were close to each other, and the tendencies of the lines connecting the mass centers were similar, indicating that the warning data used for association rule exploring were reliable. Figure 9 shows the spatial and temporal distributions of various warning types. The cumulative changes in the interaction of improper driving behaviors (eye closure and yawn) with abnormal vehicle states (lane departure, rapid

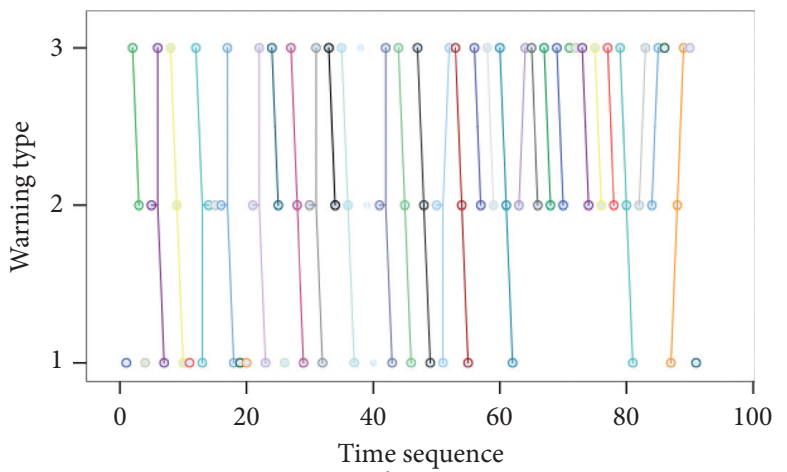

Various interaction interval

\begin{tabular}{|c|c|c|}
\hline-0 & 2.00 & 32.00 \\
\hline-0 & 8.00 & $-\circ 33.00$ \\
\hline$-\infty-$ & 9.00 & $-\infty-34.00$ \\
\hline$-\infty$ & 10.00 & $-\infty-35.00$ \\
\hline & 11.00 & -36.00 \\
\hline$-\infty$ & 13.00 & $-\infty 37.00$ \\
\hline$-\infty$ & 14.00 & $-0-39.00$ \\
\hline-0 & 15.00 & $-0-40.00$ \\
\hline$-\infty$ & 19.00 & $-0-41.00$ \\
\hline$-\infty$ & 20.00 & $-\circ-45.00$ \\
\hline$-\infty$ & 21.00 & $-0-48.00$ \\
\hline$-\infty$ & 24.00 & $-\circ 49.00$ \\
\hline$-\infty$ & 25.00 & $-\circ-50.00$ \\
\hline-0 & 27.00 & -51.00 \\
\hline$-\infty$ & 28.00 & -52.00 \\
\hline$-\infty$ & 29.00 & $-0-60.00$ \\
\hline$-\infty$ & 30.00 & -76.00 \\
\hline-0 & 31.00 & $\ldots$ \\
\hline
\end{tabular}

FIgURE 8: The interaction among lane departure, rapid acceleration, and rapid deceleration.

acceleration, and rapid deceleration) are presented in Figure 10. Despite that the number of interactions between eye closure (or yawn) and rapid acceleration (or rapid deceleration) was higher than that between eye closure (or yawn) and lane departure, the interactive tendencies of eye closure and yawn with rapid acceleration and rapid deceleration of vehicle were roughly the same, which was consistent with the results of association rules, further proving the reliability of the rules.

\section{Discussion}

In this section, we further discussed the association rules between improper driving behaviors, between abnormal 
TABLE 5: Association rules between improper driving behavior and abnormal vehicle states.

\begin{tabular}{|c|c|c|c|}
\hline Association rules & Support & Confidence & Effectivity \\
\hline Eye closure--rapid acceleration* & 0.456 & 0.476 & 0.466 \\
\hline Eye closure--rapid deceleration* & 0.410 & 0.428 & 0.419 \\
\hline Eye closure-lane departure & 0.321 & 0.335 & 0.328 \\
\hline Yawn--rapid acceleration* & 0.444 & 0.481 & 0.463 \\
\hline Yawn--rapid deceleration* & 0.375 & 0.407 & 0.391 \\
\hline Yawn-lane departure & 0.327 & 0.354 & 0.341 \\
\hline Yawn--eye closure--rapid acceleration* & 0.427 & 0.481 & 0.455 \\
\hline Yawn--eye closure--rapid deceleration* & 0.358 & 0.403 & 0.381 \\
\hline Yawn-eye closure-lane departure & 0.321 & 0.361 & 0.342 \\
\hline Yawn--lane departure--rapid acceleration* & 0.249 & 0.763 & 0.568 \\
\hline Yawn--lane departure--rapid deceleration* & 0.221 & 0.675 & 0.502 \\
\hline
\end{tabular}

Note: *indicates strong rules; otherwise, they are general rules.

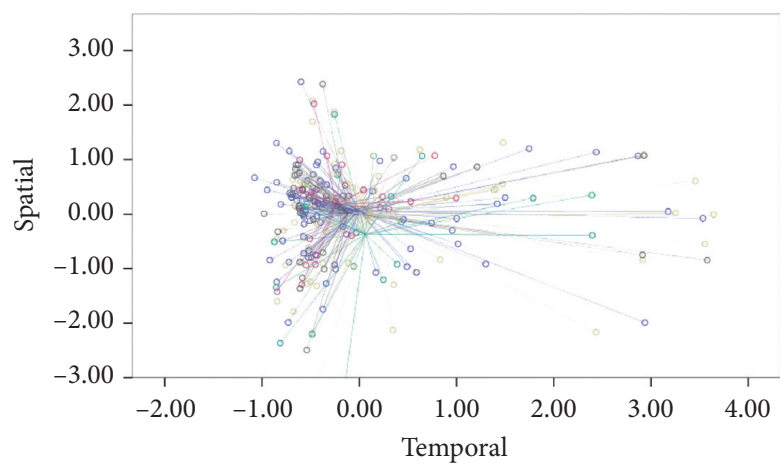

Warning type

$\begin{array}{ll}\odot \text { Eye closure } & \odot \text { Rapid acceleration } \\ \odot-\text { Lane departure } & \odot \text { Rapid deceleration } \\ - \text { Yawn } & \end{array}$

Figure 9: Spatial and temporal distributions of eye closure, yawn, lane departure, rapid acceleration, and rapid deceleration.

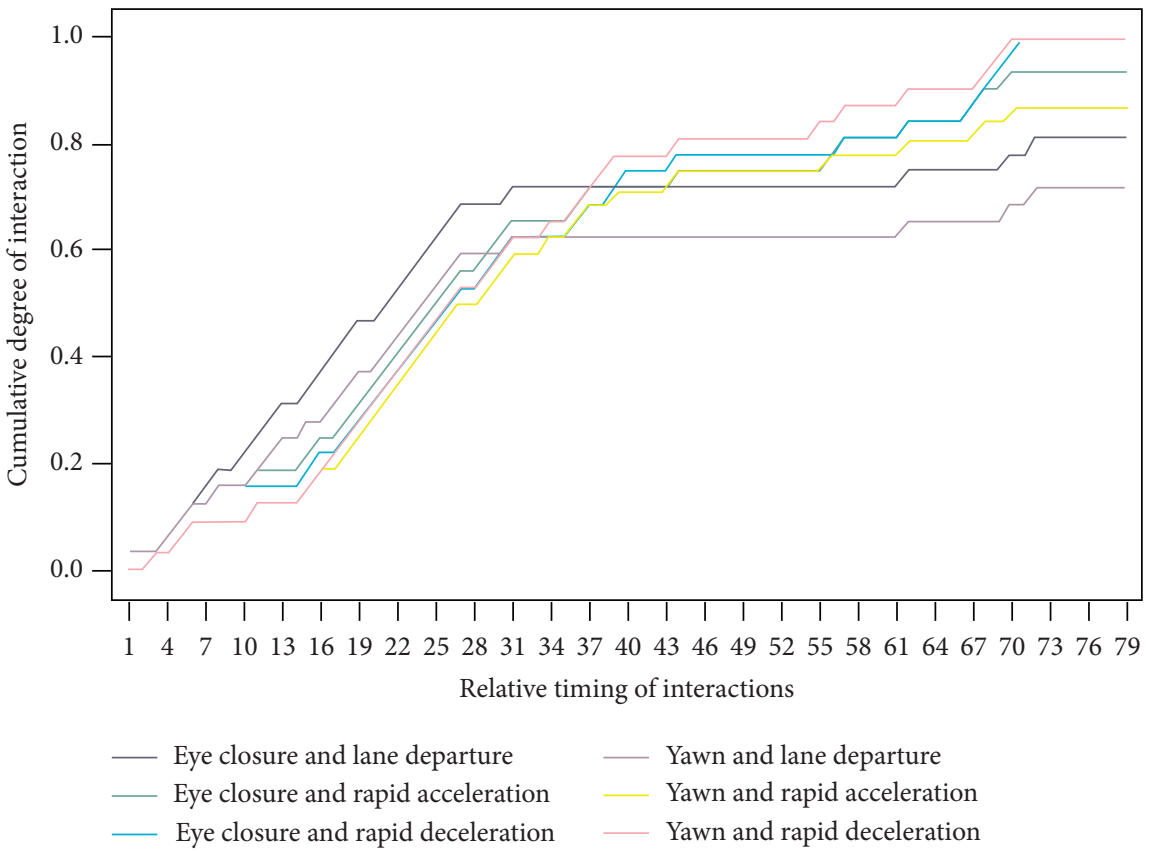

Figure 10: The accumulative changes in the interaction between improper driving behaviors and abnormal vehicle states. 
vehicle states, and between improper driving behaviors and abnormal vehicle states and explored the practical significance of the rules. Furthermore, some suggestions for optimizing the active safety control of the ADAS were proposed.

This section further discussed the association rules between improper driver behaviors, between abnormal vehicle states, and between improper driver behaviors and abnormal vehicle states, explored the practical significance of various rules, and suggested ideas for optimizing the active security control of the ADAS.

\subsection{Relationships between Improper Driving Behavior.} The exploring of association rules between improper driving behaviors is conducive to the optimization of improper behavior collection and warning mode. According to the results, the interaction between eye closure and yawn is the most frequent, and most of the interactions are caused by fatigue driving. In terms of optimizing the warning mode of the ADAS for eye closure (yawn), it is suggested to give early-warning of yawn (eye closure) or combine eye closure warning with yawn warning.

Despite that the occurrence probability of looking away was relatively low, the probability of looking away inducing eye closure and yawn was quite high. This suggests that driver's attention may not only be distracted by environmental factors (the traffic environment has an effect on driver scanning behavior, such as the effect of different intersection types on scanning behavior [27]) but also by fatigue and the complex traffic situation. Thus, apart from looking away, the DSM system should give early-warning of eye closure and yawn as well. In short, in warning the improper driving behaviors, the ADAS should give priory consideration to the association between improper driving behaviors, so as to optimize the warning mode and improve the effectiveness of the warning.

4.2. Relationships between Abnormal Vehicle States. The exploring of association rules between abnormal vehicle states can help improve the performance of vehicle in all aspects. The results show that rapid deceleration is usually accompanied by rapid acceleration (0.875) or lane departure (0.507). It can be seen that there are frequent interactions between rapid acceleration and rapid deceleration. The possible reason for that is the driver accelerates (or decelerates) out of attention distraction or response to some traffic emergencies, so the driver needs to decelerate (or accelerate) the vehicle until it returns to normal velocity.

Forward collision interacts more frequently with lane departure than with other abnormal vehicle states. This is possibly the result of drivers becoming more aggressive and focused on the area ahead after congestion [28]. It can easily cause the vehicle to trigger forward collision warning, and the driver's corresponding emergency actions often cause the vehicle to drift sideways. Prynne and Martin [29] also noted that drivers who braked and steered at the same time had the highest success rate in avoiding collisions. Therefore, the ADAS should moderately regulate the lateral deviation performance of the vehicle when there is a forward collision warning. In the present paper, the investigation on abnormal vehicle states is hope to help vehicles to be equipped with suitable intelligent driver assistance technologies so as to avoid knock-on effects of abnormal vehicle states.

\subsection{Relationships between Improper Driving Behavior and} Abnormal Vehicle States. The research on the association rules between improper driving behaviors and abnormal vehicle states contributes to enhancing driver's adaptation to the ADAS and also provides the theoretical basis for realizing the personalized interaction between driver and vehicle and for studying the man-computer interaction conflicts and personalized interaction needs in man-computer cooperative driving.

The results showed that eye closure and yawn are likely to induce abnormal states such as rapid acceleration and rapid deceleration. When the driver closes eyes and yawns, the ADAS should enhance the longitudinal control over the vehicle, especially the regulation of acceleration and deceleration. Since the effectivity of association rules of eye closure and yawn with lane departure is larger than the minimum effectivity, eye closure and yawn are very likely to induce lane departure. Therefore, besides the longitudinal control mentioned above, moderate regulation of vehicle's lateral deviation is also needed. The extent of longitudinal and lateral control over vehicle can be determined according to the probabilities of rapid acceleration, rapid deceleration, and lane departure induced by eye closure and yawn.

Rapid acceleration and deceleration are very likely to occur after yawn and lane departure. That is because the driver needs to slow down or speed up the vehicle to make it stay on the original lane after the driver yawns and the vehicle deviates from the lane. Thus, the control optimization of the ADAS should take the driver's emergency operations into account, so that the system can better assist the vehicle in emergency situations.

4.4. Practical Application of Research Results. Through these three aspects, the focus points for optimizing the ADAS and DSM system were explored. Next, how the results of this study could be applied to the ADAS would be further discussed. In this paper, the relationships between inappropriate driving behaviors and abnormal vehicle states were quantified by association rules, and the probability values (relationship occurrence probabilities) of mutual triggering and concurrency between improper driving behaviors and abnormal vehicle states were calculated. Some effective machine learning algorithms are taken based on value of the relationship occurrence probability, so that the optimization of the ADAS can be determined effort and direction. For example, based on the value of the relationship occurrence probability between various types of warnings and rapid acceleration and rapid deceleration, lane departure, the ADAS can be effectively assigned to the weight ratio of lateral and longitudinal performance in vehicles' control process. 
Also, when a certain warning occurs, based on the relationship occurrence probability and the corresponding algorithm, the system can effectively prejudge the upcoming abnormal vehicle states. This is beneficial to the effective control of the vehicle by the ADAS and avoids the lagging effect and improper prevention and control brought about by traditional prevention and control. At the same time, the concurrent probabilities of inappropriate driving behaviors and abnormal vehicle states are used to determine the multimodal fusion of warning methods, which can avoid the poor results brought about by a single method, for example, how to set warning modes from visual, auditory, and tactile perspectives. Based on the relationship occurrence probability of improper driving behaviors, determine whether multiple perspectives are considered simultaneously, from which perspectives and the weight of each perspective account for optimized alarm methods.

\section{Conclusions}

This paper explored the relationship between improver driving behavior and abnormal vehicle state under the ADAS, with the aim of providing a theoretical basis for improving driver's adaptation to the ADAS and promoting the research on the technologies regarding man-computer driving, such as the vehicle control right switching timing, interaction modes, interactive personalization, and active safety control.

The warning data collected from the driver assistance warning system installed on bus (including the warning data in forms of video, the radar warning data about the bus and its front vehicle, and the Beidou-based vehicle moving data) were cleaned and processed. Then, Person correlation analysis (the correlation coefficients were all greater than 0.9 , and the significance levels were less than 0.01.) was used to verify the correlation rules between improper driving behavior and abnormal vehicle state explored with the processed data. The results showed that the warning data of various types can be clustered. A hierarchical clustering method was used to classify the warning data into the corresponding intervals. And the clustered results and processed data were verified to be reliable and effective based on the spatial distribution of bus lines and distribution characteristics of the clustered warning items. Then, an effectivity-based improved Apriori algorithm was proposed to explore the association rules between improper driving behaviors, between abnormal vehicle states, and between improper driving behaviors and abnormal vehicle states. Subsequently, the validity of these association rules was verified with the spatial-temporal distribution characteristics and cumulative interaction curve. The results showed that eye closure and yawn were high in concurrency (probability: 0.888) and interaction (average probability: 0.946); the interaction among lane departure, rapid acceleration, and rapid deceleration are frequent (average probability: 0.7224); eye closure (average probability: 0.452 ) and yawn (average probability: 0.444 ) are likely to induce abnormal vehicle states such as rapid acceleration and rapid deceleration.
Finally, the practical significance of the association rules was discussed, and some suggestions for optimizing the active security control of the ADAS were proposed. In terms of optimizing the ADAS warnings, multiangle (visual, auditory, and tactile) fusion can be considered, e.g., eye closure and yawn warning mode for integration. In terms of the ADAS' auxiliary control of the vehicle, e.g., when eye closure or yawn occurs, the ADAS' control of the vehicle's lateral and longitudinal performance should be enhanced. Since the occurrence of forward collision is often followed by lane departure, the ADAS should be strengthened to control the vehicle's lateral drift when the vehicle has forward collision warning.

\section{Data Availability}

The data used to support the findings of this study are currently under embargo while the research findings are commercialized.

\section{Conflicts of Interest}

The authors declare no conflicts of interest.

\section{Authors' Contributions}

Y.C. undertook the collection of data, the construction of algorithmic models, the analysis of data, and the writing of the paper. T.W. provided guidance on the paper methodological ideas and interpretation of the results. T.W., X.Y., and W.L. contributed to the paper review and editing. J.C. was the supervisor of the paper.

\section{Acknowledgments}

This research was funded by the Fund for Less-Developed Regions of the National Natural Science Foundation of China (grant no. 71861006), Guangxi Natural Science Foundation (grant no. 2020GXNSFAA159153), Guangxi Science and Technology Base and Talent Special Project (grant no. AD20159035), the Natural Science Foundation of Jiangsu Province (grant no. BK20180775), the Fund of the National Natural Science Foundation of China (grant no. 61963011), the Key Project of National Natural Science Foundation of China (grant no. 51638004), and Innovation Project of GUET Graduate Education (grant no. 2020YCXS121).

\section{References}

[1] US Department of Transportation, National Highway Traffic Safety Administration, Automated Driving Systems 2.0: A Vision for Safety, U.S. Department of Transportation, National Highway Traffic Safety Administration, Washington, DC, USA, 2017.

[2] Y. F. Hu, T. Qu, J. Liu et al., "Human-machine cooperative control of intelligent vehicle: recent developments and future perspectives," Acta Automatica Sinica, vol. 45, no. 7, pp. 1261-1280, 2019.

[3] C. Z. Wu, H. R. Wu, and N. C. Lyu, "Review of control switch and safety of human-computer driving intelligent vehicle," 
Journal of Traffic and Transportation Engineering, vol. 18, no. 6, pp. 131-141, 2018.

[4] Y. Rahmati, M. K. Hosseini, A. Talebpour et al., "Influence of autonomous vehicles on car-following behavior of Human drivers," Transportation Research Record Journal of the Transportation Research Board, vol. 2673, no. 12, pp. 367-379, 2019.

[5] E. Altendorf, C. Schreck, G. Weßel, Y. Canpolat, and F. Flemisch, "Utility assessment in automated driving for cooperative human-machine systems," Cognition, Technology \& Work, vol. 21, no. 4, pp. 607-619, 2019.

[6] Q. Sun, H. Zhang, Z. Li, C. Wang, and K. Du, "ADAS acceptability improvement based on self-learning of individual driving characteristics: a case study of lane change warning system," IEEE Access, vol. 7, pp. 81370-81381, 2019.

[7] B. Zhu, Y. Shude, J. Zhao, and W. Deng, "Personalized lanechange assistance system with driver behavior identification," IEEE Transactions on Vehicular Technology, vol. 67, no. 11, pp. 10293-10306, 2018.

[8] H. L. Sang, S. Lee, and M. Kim, "Development of a driving behavior-based collision warning System using a neural network," International Journal of Automotive Technology, vol. 19, no. 5, pp. 837-844, 2018.

[9] C. Wang, R. Fu, Q. Zhang et al., "Research on parameter TTC characteristics of lane change warning System," China Journal of Highway \& Transport, vol. 28, no. 8, pp. 91-107, 2015.

[10] A. Ahmadi and S. G. Machiani, “Drivers' performance examination using a personalized adaptive curve speed warning: driving simulator study," International Journal of Human Computer Interaction, vol. 35, no. 11-15, pp. 996-1007, 2019.

[11] $\mathrm{Z}$. Yu, "Review of vehicle state estimation problem under driving situation," Journal of Mechanical Engineering, vol. 45, no. 5, pp. 20-33, 2009.

[12] P. Y. Hsiao, C. W. Yeh, S. S. Huang, and L. C. Fu, "A portable vision based real-time lane departure warning system: day and night," IEEE Transactions on Vehicular Technology, vol. 58, no. 4, pp. 2089-2094, 2009.

[13] C. Sun, C. Wu, D. Chu, M. Zhong, Z. Hu, and J. Ma, "Risk prediction for curve speed warning by considering human, vehicle, and road factors. transportation research record," Journal of the Transportation Research Board, vol. 2581, no. 1, pp. 18-26, 2016.

[14] V. E. Gai, I. V. Polyakov, M. S. Krasheninnikov et al., "Evaluation of the vehicle state with vibration-based diagnostics methods," IOP Conference Series: Materials Science and Engineering, vol. 177, Article ID 012024, 2017.

[15] E. Hashemi, M. Pirani, A. Khajepour, B. Fidan, S.-K. Chen, and B. Litkouhi, "Fault tolerant consensus for vehicle State estimation: a cyber-physical approach," IEEE Transactions on Industrial Informatics, vol. 15, no. 9, pp. 5129-5138, 2019.

[16] C. X. Song, F. Xiao, S. H. Liu, S. K. Li, L. Duan, and S. L. Peng, "State estimation of electric vehicle with in-wheel motors based on UKF," Journal of Jilin University (Engineering and Technology Edition), vol. 46, no. 2, pp. 333-339, 2016.

[17] T. Chen, L. Chen, Y. F. Cai et al., "Vehicle State fusion estimation method based on multi-model iteration," Transactions of the Chinese Society for Agricultural Machinery, vol. 49, no. 6, pp. 385-392, 2018.

[18] W. Q. Zhou, X. Qi, L. Chen et al., "Vehicle State estimation based on the combination of unscented kalman filtering and genetic algorithm," Automotive Engineering, vol. 41, no. 2, pp. 82-89, 2019.
[19] L. Li, D. Wen, N.-N. Zheng, and L.-C. Shen, "Cognitive cars: a new frontier for ADAS research," IEEE Transactions on Intelligent Transportation Systems, vol. 13, no. 1, pp. 395-407, 2012.

[20] G. Li, S. E. Li, B. Cheng, and P. Green, "Estimation of driving style in naturalistic highway traffic using maneuver transition probabilities," Transportation Research Part C: Emerging Technologies, vol. 74, pp. 113-125, 2017.

[21] X. Na and D. J. Cole, "Application of open-loop Stackelberg equilibrium to modeling a driver's interaction with vehicle active Steering control in obstacle avoidance," IEEE Transactions on Human-Machine Systems, vol. 47, no. 5, pp. 673685, 2017.

[22] Y. Zhou, M. Deng, and N. C. Lv, "An analysis of driving behaviors and safety evaluation on deceleration lane of urban arterial roads," Journal of Transport Information and Safety, vol. 37, no. 1, pp. 7-12, 2019.

[23] J. Navarro, E. Yousfi, J. Deniel, C. Jallais, M. Bueno, and A. Fort, "The impact of false warnings on partial and full lane departure warnings effectiveness and acceptance in car driving," Ergonomics, vol. 59, no. 12, pp. 1553-1564, 2016.

[24] J. M. Sullivan, M. J. Flannagan, A. K. Pradhan, and S. Bao, Literature Review of Behavioral Adaptations to Advanced Driver Assistance Systems, AAA Foundation for Traffic Safety, Washington, DC, USA, 2016.

[25] L. J. Jiang and L. Guo, "Spatial association rules mining based on taxi trajectory data," Geomatics Spatial Information Technology, vol. 47, no. 7, pp. 56-59, 2019.

[26] E. P. S. Castro, T. D. Maia, M. R. Pereira et al., "Review and comparison of Apriori algorithm implementations on hadoop-MapReduce and spark," Knowledge Engineering Review, vol. 33, 2018.

[27] G. Li, Y. Wang, F. Zhu et al., "Drivers' visual scanning behavior at signalized and unsignalized intersections: a naturalistic driving study in China," Journal of Safety Research, vol. 71, pp. 219-229, 2019.

[28] G. Li, W. Lai, X. Sui et al., "Influence of traffic congestion on driver behavior in post-congestion driving," Accident Analysis \& Prevention, vol. 141, Article ID 105508, 2020.

[29] K. Prynne and P. Martin, "Braking Behavior in emergencies," in Proceedings of the SAE Technical Paper, USA, 1995. 